N. Obata

Nagoya Math. J.

Vol. 139 (1995), 21-36

\title{
DERIVATIONS ON WHITE NOISE FUNCTIONALS
}

\author{
NOBUAKI OBATA
}

\section{Introduction}

The Gaussian space $\left(E^{*}, \mu\right)$ is a natural infinite dimensional analogue of Euclidean space with Lebesgue measure and a special choice of a Gelfand triple $(E) \subset L^{2}\left(E^{*}, \mu\right) \subset(E)^{*}$ gives a fundamental framework of white noise calculus [2] as distribution theory on Gaussian space. It is proved in Kubo-Takenaka [7] that $(E)$ is a topological algebra under pointwise multiplication. The main purpose of this paper is to answer the fundamental question: what are the derivations on the algebra $(E)$ ?

Since $(E)$ is a topological algebra, each $\Phi \in(E)^{*}$ gives rise to a multiplication operator $\phi \mapsto \Phi \phi=\phi \Phi \in(E)^{*}, \phi \in(E)$. In fact, this is a continuous operator, namely, $\Phi \in \mathscr{L}\left((E),(E)^{*}\right)$. We then adopt a slightly general definition: a linear operator $E$ from $(E)$ into $(E)^{*}$ is called a derivation if

$$
\Xi(\phi \psi)=\Xi \phi \cdot \phi+\phi \cdot \Xi \phi, \quad \phi, \phi \in(E) .
$$

In this paper we determine all continuous derivations on $(E)$; more precisely, the derivations which belong to $\mathscr{L}\left((E),(E)^{*}\right)$ or $\mathscr{L}((E),(E))$. The main result is stated in Theorem 5.1 .

As a result, we shall see that a continuous derivation is nothing but a first order differential operator with distribution coefficients. Its formal expression is given as

$$
\Xi=\int_{T} \Phi_{t} \partial_{t} d t
$$

where $\partial_{t}$ is Hida's differential operator, i.e., an annihilation operator at a point $t \in T$, and $\Phi_{t} \in(E)^{*}$ is (identified with) a multiplication operator with parameter $t \in T$. In fact, $t \mapsto \Phi_{t}$ is an $(E)^{*}$-valued distribution on $T$, namely, $\tilde{\Phi}(t, x)=$ $\Phi_{t}(x)$ is an element in $E_{\mathbf{C}}^{*} \otimes(E)^{*} \cong\left(E_{\mathbf{C}} \otimes(E)\right)^{*}$, for the rigorous definition see Section 3. Moreover, the operator $\Xi$ defined as in (0-2) belongs to $\mathscr{L}((E),(E))$ if

Received June 21, 1993. 
and only if $\tilde{\Phi} \in E_{\mathbf{C}}^{*} \otimes(E)$, namely, if and only if $E$ is a first order differential operator with smooth coefficients.

The discussion is based on the theory of Fock expansion of operators on white noise functionals established in a series of works [12], [13], [14]. The essence of this theory lies in the fact that every operator $E \in \mathscr{L}\left((E),(E)^{*}\right)$ admits an infinite series expansion in terms of integral kernel operators, see also Section 4. For another application of this effective theory, see e.g., [11].

There has been observed formal analogy between white noise calculus and the calculus on Euclidean space based on the Gelfand triple $\&\left(\mathbf{R}^{n}\right) \subset L^{2}\left(\mathbf{R}^{n}\right) \subset$ $\mathscr{S}^{*}\left(\mathbf{R}^{n}\right)$, e.g., rotation groups, Laplacians, Fourier transform, see [3], [4], [9], [11]. A more informative expression of $(0-2)$ would be

$$
\Xi \phi(x)=\int_{T} \Phi_{t}(x) \partial_{t} \phi(x) d t, \quad \phi \in(E), x \in E^{*} .
$$

We then easily understand that the operator $E$ is a white noise analogue of a usual first order differential operator on Euclidean space given as

$$
D \phi(x)=\sum_{j=1}^{n} A_{j}(x) \frac{\partial \phi}{\partial x_{j}}(x), \quad \phi \in \&\left(\mathbf{R}^{n}\right), x=\left(x_{1}, \cdots, x_{n}\right) \in \mathbf{R}^{n} .
$$

Thus the formal analogy is again reinforced with our result in this paper.

Obviously, a first order differential operator on $E^{*}$ gives rise to a vector field on it and vice versa. It is then interesting to investigate a (local) one-parameter group of tranformations on $E^{*}$ which generates the vector field, for a particular case see [4]. As a next step, it will be interesting to discuss an operator of the form:

$$
\int_{T \times T} \partial_{s}^{*} \Phi_{s, t}(x) \partial_{t} d s d t
$$

which gives rise to a quadratic form on $(E)$ in a natural way. In [5] a simple case $\Phi_{s, t}(x)=\tau(s, t) \Phi(x)$ is discussed in relation with Dirichlet forms on white noise functionals. Furthermore, there are similarities between the above mentioned operators and quantum stochastic integrals, see e.g., [10], [15]. In fact, $(0-2)$ and $(0-3)$ are considered as direct generalizations of quantum stochastic integrals against the annihilation process and the number process, respectively. Systematic approaches to those topics will be carried out elsewhere. 


\section{White noise functionals}

To avoid tedious introduction of notation we use the same framework as settled in [4] under the name of standard setup of white noise calculus. Nevertheless we recapitulate minimal notation for readers' convenience, for details see also [12], [13], [14], etc.

Let $T$ be a topological space equipped with a Borel measure $d \nu(t)=d t$ and introduce a real Hilbert space $H=L^{2}(T, \nu ; \mathbf{R})$ with norm $|\cdot|_{0}$ and inner product $\langle\cdot, \cdot\rangle$. Let $A$ be a positive selfadjoint operator on $H$ with Hilbert-Schmidt inverse and assume that inf $\operatorname{Spec}(A)>1$. Then there exist an increasing sequence of positive numbers $1<\lambda_{0} \leq \lambda_{1} \leq \lambda_{2} \leq \cdots$ and a complete orthonormal basis $\left\{e_{j}\right\}_{j=0}^{\infty}$ for $H$ such that $A e_{j}=\lambda_{j} e_{j}$. We use the following constant numbers:

$$
\delta \equiv\left\|A^{-1}\right\|_{\mathrm{HS}}=\left(\sum_{j=0}^{\infty} \lambda_{j}^{-2}\right)^{1 / 2}<\infty, \quad 0<\rho \equiv\left\|A^{-1}\right\|_{\mathrm{OP}}=\lambda_{0}^{-1}<1 .
$$

Then a Gelfand triple $E \subset H \subset E^{*}$ is constructed in the standard manner, where the nuclear Fréchet space $E$ is equipped with Hilbertian norms:

$$
|\xi|_{p}=\left|A^{p} \xi\right|_{0}=\left(\sum_{j=0}^{\infty} \lambda_{j}^{2 p}\left\langle\xi, e_{j}\right\rangle^{2}\right)^{1 / 2}, \quad \xi \in E, \quad p \in \mathbf{R} .
$$

The canonical bilinear form on $E^{*} \times E$ is also denoted by $\langle\cdot, \cdot\rangle$. Hereafter we assume the usual conditions (H1)-(H3) to keep a delta function $\delta_{t}$ in $E^{*}$, see [4], [14].

The Gaussian space is by definition the probability space $\left(E^{*}, \mu\right)$, where $\mu$ is the Gaussian measure defined by

$$
\exp \left(-\frac{1}{2}|\xi|_{0}^{2}\right)=\int_{E^{*}} e^{i\langle x, \xi\rangle} \mu(d x), \quad \xi \in E .
$$

The Wiener-Itô-Segal isomorphism between $\left(L^{2}\right) \equiv L^{2}\left(E^{*}, \mu ; \mathbf{C}\right)$ and the Boson Fock space over $H_{\mathbf{C}}$ is given by means of the Wick ordered product as

$$
\phi(x)=\sum_{n=0}^{\infty}\left\langle: x^{\otimes_{n}}:, f_{n}\right\rangle, \quad x \in E^{*},
$$

where $\phi \in\left(L^{2}\right)$ and $f_{n} \in H_{\mathbf{C}}^{\otimes}$. Note also that each $\left\langle: x^{\otimes n}:, f_{n}\right\rangle$ is defined as an $L^{2}$-function and that the series is an orthogonal direct sum.

For $\phi \in\left(L^{2}\right)$ given as in (1-1) the second quantized operator $\Gamma(A)$ acts as

$$
(\Gamma(A) \phi)(x)=\sum_{n=0}^{\infty}\left\langle: x^{\otimes_{n}}:, A^{\otimes_{n}} f_{n}\right\rangle .
$$


With the maximal domain $\Gamma(A)$ becomes a positive selfadjoint operator with Hilbert-Schmidt inverse and thereby we obtain a complex Gelfand triple:

$$
(E) \subset\left(L^{2}\right)=L^{2}\left(E^{*}, \mu ; \mathbf{C}\right) \subset(E)^{*} .
$$

Here $(E)$ is again a nuclear Fréchet space equipped with Hilbertian norms:

$$
\|\phi\|_{p}^{2}=\left\|\Gamma(A)^{p} \phi\right\|_{0}^{2}=\sum_{n=0}^{\infty} n !\left|\left(A^{\otimes n}\right)^{p} f_{n}\right|_{0}^{2}=\sum_{n=0}^{\infty} n !\left|f_{n}\right|_{p}^{2}, \quad p \in \mathbf{R},
$$

where $\phi \in(E)$ and $f_{n} \in H_{\mathbf{C}}^{\otimes_{n}}$ are related as in (1-1) and $\|\cdot\|_{0}$ denotes the norm of $\left(L^{2}\right)$. In particular, if $\phi \in(E)$ then $f_{n} \in H_{\mathbf{C}}^{\otimes_{n}}$ for all $n=0,1,2, \cdots$. Elements in $(E)$ and $(E)^{*}$ are called a test (white noise) functional and a generalized (white noise) functional, respectively. We denote by $\langle\cdot \cdot, \cdot\rangle\rangle$ the canonical bilinear form on $(E)^{*} \times(E)$.

By construction each $\phi \in(E)$ is a function on $E^{*}$ determined only up to $\mu$-null functions. Kubo-Yokoi's continuous version theorem [8] asserts that for $\phi \in(E)$ the series $(1-1)$ converges absolutely at each $x \in E^{*}$ and becomes a unique continuous function on $E^{*}$ which coincides with $\phi$ up to $\mu$-null functions Hereafter we always assume that $(E)$ consists of such continuous functions on $E^{*}$.

As for generalized white noise functionals similar but formal expression as in $(1-1)$ is also possible and useful in many applications.

\section{Gradient operator}

We first recall basic differential operators on white noise functionals. Let $\phi \in(E)$ be given as

$$
\phi(x)=\sum_{n=0}^{\infty}\left\langle: x^{\otimes n}:, f_{n}\right\rangle, \quad x \in E^{*}, \quad f_{n} \in E_{\mathbf{C}}^{\bar{\otimes} n} .
$$

Then for any $y \in E^{*}$ we put

$$
\begin{aligned}
D_{y} \phi(x) & =\lim _{\theta \rightarrow 0} \frac{\phi(x+\theta y)-\phi(x)}{\theta} \\
& =\sum_{n=1}^{\infty} n\left\langle: x^{\otimes(n-1)}:, y \bigotimes_{1} f_{n}\right\rangle, \quad x \in E^{*}, \quad \phi \in(E),
\end{aligned}
$$

where $\bigotimes_{1}$ stands for the contraction of tensor products. The limit always exists and the series converges absolutely as numerical series. Moreover, it is known [4] that for any $p \geq 0$ and $q>0$, 


$$
\left\|D_{y} \phi\right\|_{p} \leq\left(\frac{\rho^{-2 q}}{-2 e q \log \rho}\right)^{1 / 2}|y|_{-(p+q)}\|\phi\|_{p+q}, \quad \phi \in(E)
$$

In particular, $D_{y} \in \mathscr{L}((E),(E))$. Hida's differential operator is defined as $\partial_{t}=D_{\delta_{t}}$, where $\delta_{t} \in E^{*}$ is the delta function at $t \in T$. It is nothing but an annihilation operator at a point $t \in T$ in usual Fock space language.

It is convenient to use a white noise analogy of the gradient. We put

$$
\nabla \phi(t, x)=\partial_{t} \phi(x), \quad t \in T, \quad x \in E^{*} .
$$

This operator is well known in various contexts, see e.g., [1], [5], [6]. For further discussion we need $E_{\mathbf{C}} \otimes(E)$, i.e., the space of $E_{\mathbf{C}}$-valued white noise test functionals. As usual the symbol $\otimes$ stands for the completed $\pi$-tensor product following our convention [4], [14]. It is known (see e.g., [13]) that the topology of $E_{\mathbf{C}} \otimes$ $(E)$ is given by the norms

$$
\|\omega\|_{p}=\left\|(A \otimes \Gamma(A))^{p} \omega\right\|_{0}, \quad \omega \in E_{\mathbf{C}} \otimes(E), \quad p \in \mathbf{R} .
$$

With these notation we prove the following

Proposition 2.1. It holds that

$$
\nabla \phi=\sum_{j=0}^{\infty} e_{j} \otimes D_{e_{j}} \phi, \quad \phi \in(E),
$$

where the series converges in $E_{\mathbf{C}} \otimes(E)$ as well as pointwisely. Moreover, for any $p \geq 0$

$$
\|\nabla \phi\|_{p}^{2}=\sum_{j=0}^{\infty}\left\|e_{j} \otimes D_{e_{j}} \phi\right\|_{p}^{2} \leq\left(\frac{\rho^{-2} \delta^{2}}{-2 e \log \rho}\right)\|\phi\|_{p+1}^{2}, \quad \phi \in(E) .
$$

In particular, $\nabla \in \mathscr{L}\left((E), E_{\mathbf{C}} \otimes(E)\right)$.

Proof. Suppose that $\phi \in(E)$ is given as in $(2-1)$. Then, by definition

$$
\nabla \phi(t, x)=\partial_{t} \phi(x)=\sum_{n=1}^{\infty} n\left\langle: x^{\otimes(n-1)}:, \delta_{t} \otimes_{1} f_{n}\right\rangle .
$$

Using the Fourier expansion of $f_{n}$ in terms of $\left\{e_{j}\right\}_{j=0}^{\infty}$, we obtain

$$
\nabla \phi(t, x)=\sum_{j=0}^{\infty} \sum_{n=1}^{\infty} n\left\langle: x^{\otimes(n-1)}:, e_{j} \bigotimes_{1} f_{n}\right\rangle e_{j}(t)
$$

For simplicity we put $\phi_{j}=D_{e_{j}} \phi$. Then, by (2-2) 


$$
\nabla \phi(t, x)=\sum_{j=0}^{\infty} e_{j}(t) \phi_{j}(x)
$$

As is easily verified, the above infinite series (2-8), (2-9) and (2-10) converge absolutely at each $t \in T$ and $x \in E^{*}$.

We next investigate a norm estimate. In view of (2-3) we have

$$
\left\|\phi_{j}\right\|_{p} \leq\left(\frac{\rho^{-2 q}}{-2 e q \log \rho}\right)^{1 / 2}\left|e_{j}\right|_{-(p+q)}\|\phi\|_{p+q}, \quad p \geq 0, \quad q>0 .
$$

On the other hand, since $\left\{e_{j}\right\}_{j=0}^{\infty}$ is an orthogonal set with respect to every norm $|\cdot|_{p}$, we see from $(2-5)$ and $(2-10)$ that

$$
\|\nabla \phi\|_{p}^{2}=\left\|(A \otimes \Gamma(A))^{p} \nabla \phi\right\|_{0}^{2}=\sum_{j=0}^{\infty}\left|e_{j}\right|_{p}^{2}\left\|\phi_{j}\right\|_{p}^{2}=\sum_{j=0}^{\infty}\left\|e_{j} \otimes \phi_{j}\right\|_{p}^{2},
$$

which proves the first half of $(2-7)$. Inserting $(2-11)$ into $(2-12)$ we obtain

$$
\|\nabla \phi\|_{p}^{2}=\sum_{j=0}^{\infty}\left|e_{j}\right|_{p}^{2}\left(\frac{\rho^{-2 q}}{-2 e q \log \rho}\right)\left|e_{j}\right|_{-(p+q)}^{2}\|\phi\|_{p+q}^{2}=\left(\frac{\rho^{-2 q}}{-2 e q \log \rho}\right)\|\phi\|_{p+q}^{2} \sum_{j=0}^{\infty} \lambda_{j}^{-2 q} .
$$

Thus the second half of $(2-7)$ follows. by taking $q=1$. The rest of the assertion is now immediate.

Q,E.D.

Corollary 2.2. For $y \in E^{*}$ and $\Phi \in(E)^{*}$ it holds that

$$
\langle y \otimes \Phi, \nabla \phi\rangle=\left\langle\left\langle\Phi, D_{y} \phi\right\rangle, \quad \phi \in(E) .\right.
$$

Here the canonical bilinear form on $\left(E_{\mathbf{C}} \otimes(E)\right)^{*} \times\left(E_{\mathbf{C}} \otimes(E)\right)$ is also denoted by $\langle\langle\cdot, \cdot\rangle$. It is also possible to adopt $(2-13)$ as the definition of $\nabla \phi$.

\section{First order differential operators}

Before going into the definition of an operator of the form (0-2) we recall the following

Lemma 3.1 ([7]). For each $p \geq 0$ there exist $q>0$ and $C \geq 0$ such that

$$
\|\phi \phi\|_{p} \leq C\|\phi\|_{p+q}\|\phi\|_{p+q}, \quad \phi, \phi \in(E) .
$$

In this paper we do not need a precise norm estimate though it is very interesting in itself, see e.g., [14]. We next prove the following 
Lemma 3.2. For $\phi, \phi \in(E)$ put

$$
\omega_{\phi, \phi}(t, x)=\left(\partial_{t} \phi\right)(x) \cdot \psi(x), \quad t \in T, \quad x \in E^{*} .
$$

Then, $\omega_{\phi, \psi} \in E \otimes(E)$. Moreover, $(\phi, \phi) \mapsto \omega_{\phi, \psi}$ is a continuous bilinear map from $(E) \times(E)$ into $E_{\mathbf{C}} \otimes(E)$.

Proof. For simplicity we write $\omega=\omega_{\phi, \phi}$. It then follows from Proposition 2.1 that

$$
\omega(t, x)=\nabla \phi(t, x) \cdot \phi(x)=\sum_{j=0}^{\infty} e_{j}(t) \phi_{j}(x) \phi(x), \quad t \in T, \quad x \in E^{*},
$$

where $\phi_{j}=D_{e_{j}} \phi$. Suppose $p \geq 0$ is given. Then, in view of Lemma 3.1,

$$
\|\omega\|_{p}^{2}=\sum_{j=0}^{\infty}\left|e_{j}\right|_{p}^{2}\left\|\phi_{j} \phi\right\|_{p}^{2} \leq C^{2}\|\phi\|_{p+q}^{2} \sum_{j=0}^{\infty}\left|e_{j}\right|_{p}^{2}\|\phi\|_{p+q}^{2},
$$

for some $C \geq 0$ and $q>0$. Using $\left|e_{j}\right|_{p}=\rho^{q}\left|e_{j}\right|_{p+q}$, we obtain

$$
\|\omega\|_{p}^{2} \leq C^{2} \rho^{2 q}\|\phi\|_{p+q}^{2} \sum_{j=0}^{\infty}\left|e_{j}\right|_{p+q}^{2}\left\|\phi_{j}\right\|_{p+q}^{2}
$$

and therefore, by $(2-7)$ we come to

$$
\left\|\omega_{\phi, \psi}\right\|_{p} \leq M\|\phi\|_{p+q+1}\|\phi\|_{p+q}, \quad \phi, \phi \in(E),
$$

where $M=C \rho^{q-1} \delta(-2 e \log \rho)^{-1 / 2}$. This completes the proof.

Q.E.D.

TheOREm 3.3. For $\tilde{\Phi} \in\left(E_{\mathbf{C}} \otimes(E)\right)^{*}$ there exists a unique operator $\Xi \in$ $\mathscr{L}\left((E),(E)^{*}\right)$ such that

$$
\langle\Xi \phi, \phi\rangle=\left\langle\left\langle\tilde{\Phi}, \omega_{\phi, \psi}\right\rangle, \quad \phi, \phi \in(E),\right.
$$

where $\omega_{\phi, \psi}$ is defined as in $(3-2)$.

Proof. Choose $p \geq 0$ such as $\|\tilde{\Phi}\|_{-p}<\infty$. Then, by (3-4) we have

$$
\left|\left\langle\tilde{\Phi}, \omega_{\phi, \phi}\right\rangle\right| \leq\|\tilde{\Phi}\|_{-p}\left\|\omega_{\phi, \phi}\right\|_{p} \leq M\|\tilde{\Phi}\|_{-p}\|\phi\|_{p+q+1}\|\phi\|_{p+q}
$$

for some $q>0$ and $M \geq 0$. This means that $(\phi, \phi) \mapsto\left\langle\tilde{\Phi}, \omega_{\phi, \phi}\right\rangle$ is a continuous bilinear form on $(E) \times(E)$, and therefore there exists a unique operator $\Xi \in$ $\mathscr{L}\left((E),(E)^{*}\right)$ satisfying $(3-5)$.

Q.E.D. 
The above constructed operator $\Xi$ is called a first order differential operator with coefficient $\tilde{\Phi} \in\left(E_{\mathbf{C}} \otimes(E)\right)^{*}$ and is denoted (somehow formally) by

$$
\Xi=\int_{T} \Phi_{t} \partial_{t} d t
$$

Here we write $\Phi_{t}(x)=\tilde{\Phi}(t, x)$. In fact $t \mapsto \Phi_{t}$ is an $(E)^{*}$-valued distribution on $T$, namely, an element in $E_{\mathbf{C}}^{*} \otimes(E)^{*} \cong\left(E_{\mathbf{C}} \otimes(E)\right)^{*}$.

We are now interested in first order differential operators acting on $(E)$ into itself.

THEOREM 3.4. Let $\Xi$ be a first order differential operator with coefficient $\tilde{\Phi} \in$ $\left(E_{\mathbf{C}} \otimes(E)\right)^{*}$. Then $\Xi \in \mathscr{L}((E),(E))$ if and only if $\tilde{\Phi} \in E_{\mathbf{C}}^{*} \otimes(E)$.

Proof. There is a canonical isomorphism $\left(E_{\mathbf{C}} \otimes(E)\right)^{*} \cong \mathscr{L}\left(E_{\mathbf{C}},(E)^{*}\right)$ : the correspondence between $\tilde{\Phi} \in\left(E_{\mathbf{C}} \otimes(E)\right)^{*}$ and $K \in \mathscr{L}\left(E_{\mathbf{C}},(E)^{*}\right)$ is given by

$$
\left\langle\langle\tilde{\Phi}, \xi \otimes \phi\rangle=\left\langle\langle K \xi, \phi\rangle, \quad \xi \in E_{\mathbf{C}}, \quad \phi \in(E) .\right.\right.
$$

Under this isomorphism, $\tilde{\Phi} \in E_{\mathbf{C}}^{*} \otimes(E)$ if and only if $K \in \mathscr{L}\left(E_{\mathbf{C}},(E)\right)$.

Suppose that $\boldsymbol{E}$ is given as in (3-6). Then, by definition (3-5) holds. On the other hand, it has been established during the proof of Lemma 3.2 that

$$
\omega_{\phi, \psi}=\sum_{j=0}^{\infty} e_{j} \otimes\left(\phi_{j} \phi\right), \quad \phi_{j}=D_{e_{j}} \phi
$$

converges in $E_{\mathbf{C}} \otimes(E)$. Then (3-5) becomes

$$
\langle\Xi \Xi \phi, \phi\rangle\rangle=\sum_{j=0}^{\infty}\left\langle\left\langle\tilde{\Phi}, e_{j} \otimes\left(\phi_{j} \phi\right)\right\rangle=\sum_{j=0}^{\infty}\left\langle\left\langle K e_{j}, \phi_{j} \psi\right\rangle\right\rangle=\sum_{j=0}^{\infty}\left\langle\left\langle\phi_{j} K e_{j}, \phi\right\rangle .\right.\right.
$$

Hence for any $p \geq 0$,

$$
|\langle\Xi \phi, \phi\rangle| \leq \sum_{j=0}^{\infty}\left\|\phi_{j} K e_{j}\right\|_{p}\|\phi\|_{-p},
$$

though the sum is possibly infinite. We now suppose that $\tilde{\Phi} \in E_{\mathbf{C}}^{*} \otimes(E)$, or equivalently, $K \in \mathscr{L}\left(E_{\mathbf{C}},(E)\right)$. Then $K e_{j} \in(E)$ and by Lemma 3.1 there exist $q>0$ and $C_{1} \geq 0$ such that

$$
\left\|\phi_{j} K e_{j}\right\|_{p} \leq C_{1}\left\|\phi_{j}\right\|_{p+q}\left\|K e_{j}\right\|_{p+q} .
$$

Moreover, there exist $r \geq 0$ and $C_{2} \geq 0$ such that 


$$
\left\|K e_{j}\right\|_{p+q} \leq C_{2}\left|e_{j}\right|_{p+q+r}
$$

Thus (3-8) becomes

$$
\begin{aligned}
|\langle\Xi \phi, \phi\rangle| & \leq C_{1} C_{2}\|\phi\|_{-p} \sum_{j=0}^{\infty}\left\|\phi_{j}\right\|_{p+q}\left|e_{j}\right|_{p+q+r} \\
& =C_{1} C_{2}\|\phi\|_{-p} \sum_{j=0}^{\infty}\left\|\phi_{j}\right\|_{p+q}\left|e_{j}\right|_{p+q+r+1} \lambda_{j}^{-1} \\
& \leq C_{1} C_{2}\|\phi\|_{-p}\left(\sum_{j=0}^{\infty}\left\|\phi_{j}\right\|_{p+q}^{2}\left|e_{j}\right|_{p+q+r+1}^{2}\right)^{1 / 2}\left(\sum_{j=0}^{\infty} \lambda_{j}^{-2}\right)^{1 / 2} \\
& \leq C_{1} C_{2} \delta\|\phi\|_{-p}\left(\sum_{j=0}^{\infty}\left\|e_{j} \otimes \phi_{j}\right\|_{p+q+r+1}^{2}\right)^{1 / 2} .
\end{aligned}
$$

It then follows from Proposition 2.1 that

$$
|\langle\Xi \phi, \phi\rangle| \leq C_{1} C_{2} \delta\|\phi\|_{-p}\|\nabla \phi\|_{p+q+r+1},
$$

and hence

$$
\|\Xi \phi\|_{p} \leq C_{1} C_{2} \delta\|\nabla \phi\|_{p+q+r+1} \leq C_{1} C_{2} \delta\left(\frac{\rho^{-2} \delta^{2}}{-2 e \log \rho}\right)^{1 / 2}\|\phi\|_{p+q+r+2} .
$$

We have thus seen that $\Xi \in \mathscr{L}((E),(E))$.

Conversely, suppose that $\Xi \in \mathscr{L}((E),(E))$. Then for any $p \geq 0$ there exist $q$ $\geq 0$ and $C \geq 0$ such that

$$
\|\Xi \phi\|_{p} \leq C\|\phi\|_{p+q}, \quad \phi \in(E) .
$$

Let $\xi \in E_{\mathbf{C}}$ be fixed and consider

$$
\phi(x)=\langle x, \xi\rangle, \quad x \in E^{*} .
$$

As is easily verified, $\omega_{\phi, \psi}=\xi \otimes \phi$ for any $\phi \in(E)$. Hence by (3-5) and (3-7) we obtain

$$
\langle\Xi \phi, \phi\rangle=\left\langle\tilde{\Phi}, \omega_{\phi, \phi}\right\rangle=\langle\tilde{\Phi}, \xi \otimes \psi\rangle=\langle K \xi, \phi\rangle .
$$

Then by (3-9) we obtain

$$
|\langle K \xi, \phi\rangle|=|\langle\Xi \phi, \phi\rangle| \leq\|\Xi \phi\|_{p}\|\phi\|_{-p} \leq C\|\phi\|_{p+q}\|\phi\|_{-p} .
$$

Therefore,

$$
\|K \xi\|_{p} \leq C\|\phi\|_{p+q}=C|\xi|_{p+q}, \quad \xi \in E_{\mathbf{C}} .
$$

Consequently, $K \in \mathscr{L}\left(E_{\mathbf{C}},(E)\right)$

Q.E.D. 
Such an operator $E$ as described in Theorem 3.4 is called a first order differential operator with smooth coefficients. This would be reasonable because in that case $t \mapsto \Phi_{t}$ is an $(E)$-valued distribution on $T$.

\section{Integral kernel operators and Fock expansion}

With each $\kappa \in\left(E_{\mathbf{C}}^{\otimes(l+m)}\right)^{*}$ we may associate an integral kernel operator whose formal expression is given by

$$
\Xi_{l, m}(\kappa)=\int_{T^{l+m}} \kappa\left(s_{1}, \cdots, s_{l}, t_{1}, \cdots, t_{m}\right) \partial_{s_{1}}^{*} \cdots \partial_{s_{l}}^{*} \partial_{t_{1}} \cdots \partial_{t_{m}} d s_{1} \cdots d s_{l} d t_{1} \cdots d t_{m},
$$

where $\kappa$ is called the kernel distribution. More precisely, it is defined through two canonical bilinear forms:

$$
\left\langle\left\langle\Xi_{l, m}(\kappa) \phi, \phi\right\rangle\right\rangle=\left\langle\kappa,\left\langle\left\langle\partial_{s_{1}}^{*} \cdots \partial_{s_{l}}^{*} \partial_{t_{1}} \cdots \partial_{t_{m}} \phi, \phi\right\rangle\right\rangle, \phi, \phi \in(E) .\right.
$$

It is proved that $\Xi_{l, m}(\kappa) \in \mathscr{L}\left((E),(E)^{*}\right)$, see [4] for further details. Without loss of generality we may assume that the kernel distribution $\kappa$ is symmetric with respect to the first $l$ and the last $m$ variables independently. We denote by $\left(E_{\mathbf{C}}^{\otimes(l+m)}\right)_{\text {sym }(l, m)}^{*}$ the space of such $\kappa \in\left(E_{\mathbf{C}}^{\otimes(l+m)}\right)^{*}$.

THEOREM 4.1 ([12]). For any $\Xi \in \mathscr{L}\left((E),(E)^{*}\right)$ there exists a unique family of distributions $\kappa_{l, m} \in\left(E_{\mathbf{C}}^{\otimes(l+m)}\right)_{\mathrm{sym}(l, m)}^{*}$ such that

$$
\Xi \phi=\sum_{l, m=0}^{\infty} \Xi_{l, m}\left(\kappa_{l, m}\right) \phi, \quad \phi \in(E)
$$

where the right hand side converges in $(E)^{*}$.

More complete results are found in [14]. The unique expression of $\Xi \in$ $\mathscr{L}\left((E),(E)^{*}\right)$ given in Theorem 4.1 is called the Fock expansion of $\Xi$ and denoted simply by

$$
\Xi=\sum_{l, m=0}^{\infty} \Xi_{l, m}\left(\kappa_{l, m}\right)
$$

It is also known that the series converges in $\mathscr{L}\left((E),(E)^{*}\right)$.

Given $\Xi \in \mathscr{L}\left((E),(E)^{*}\right)$, the kernel distributions $\kappa_{l, m}$ are easily found by using an operator symbol. For each $\xi \in E_{\mathbf{C}}$ the exponential vector $\phi_{\xi} \in(E)$ is defined by 


$$
\phi_{\xi}(x)=\exp \left(\langle x, \xi\rangle-\frac{1}{2}\langle\xi, \xi\rangle\right)=\sum_{n=0}^{\infty}\left\langle: x^{\otimes n}: \frac{\xi^{\otimes n}}{n !}\right\rangle, \quad x \in E^{*}
$$

For $\Xi \in \mathscr{L}\left((E),(E)^{*}\right)$ a function on $E_{\mathbf{C}} \times E_{\mathbf{C}}$ defined by

$$
\hat{\Xi}(\xi, \eta)=\left\langle\left\langle\Xi \phi_{\xi}, \phi_{\eta}\right\rangle, \quad \xi, \eta \in E_{\mathbf{C}},\right.
$$

is called the symbol of $\Xi$. For example, for $\Xi$ with Fock expansion (4-2) we have

$$
e^{-\langle\xi, \eta\rangle} \hat{\Xi}(\xi, \eta)=\sum_{l, m=0}^{\infty}\left\langle\kappa_{l, m}, \eta^{\otimes l} \otimes \xi^{\otimes m}\right\rangle, \quad \xi, \eta \in E_{\mathbf{C}}
$$

Hence, in order to find kernel distributions $\kappa_{l, m}$ one needs only to consider the Taylor expansion of (4-5).

We also note the following

Proposition 4.2. For a first order differential operator $\Xi$ with coefficient $\tilde{\Phi} \in\left(E_{\mathbf{C}} \otimes(E)\right)^{*}$ we have

$$
e^{-\langle\xi, \eta\rangle} \hat{\Xi}(\xi, \eta)=\left\langle\tilde{\Phi}, \xi \otimes \phi_{\xi+\eta}\right\rangle, \quad \xi, \eta \in E_{\mathbf{C}} .
$$

Proof. By definition (3-2) we have

$$
\omega_{\phi_{\xi}, \phi_{\eta}}(t, x)=\partial_{t} \phi_{\xi}(x) \phi_{\eta}(x)=\xi(t) \phi_{\xi}(x) \phi_{\eta}(x), \quad \xi, \eta \in E_{\mathbf{C}},
$$

namely, $\omega_{\phi_{\xi}, \phi_{\eta}}=e^{\langle\xi, \eta\rangle} \xi \otimes \phi_{\xi+\eta}$. Then

$$
\tilde{\Xi}(\xi, \eta)=\left\langle\left\langle\Xi \phi_{\xi}, \phi_{\eta}\right\rangle\right\rangle=\left\langle\left\langle\tilde{\Phi}, \omega_{\phi_{\xi}, \phi_{\eta}}\right\rangle\right\rangle=e^{\langle\xi, \eta\rangle}\left\langle\left\langle\tilde{\Phi}, \xi \otimes \phi_{\xi+\eta}\right\rangle\right\rangle .
$$

This shows (4-6).

Q.E.D.

Corollary 4.3. Let $\kappa \in E_{\mathbf{C}}^{*}$ and put $\tilde{\Phi}=\kappa \otimes 1 \in E_{\mathbf{C}}^{*} \otimes(E)$. Then the first order differential operator with coefficient $\tilde{\Phi}$ coincides with $\Xi_{0,1}(\kappa)$.

Such an operator described as in Corollary 4.3 is called a first order differential operator with constant coefficients.

\section{Main result}

Recall that a linear operator $E:(E) \rightarrow(E)^{*}$ is called a derivation if

$$
\Xi(\phi \psi)=\Xi \phi \cdot \phi+\phi \cdot \Xi \phi, \quad \phi, \phi \in(E) .
$$

We then come to the main result. 
Theorem 5.1. Any continuous derivation in $\mathscr{L}\left((E),(E)^{*}\right)$ is a first order differential operator and vice versa. Furthermore, any continuous derivation in $\mathscr{L}((E)$, $(E))$ is a first order differential operator with smooth coefficients and vice versa.

For the proof we prepare a few lemmas.

Lemma 5.2. Let $\Xi \in \mathscr{L}\left((E),(E)^{*}\right)$. Then, it is a derivation if and only if

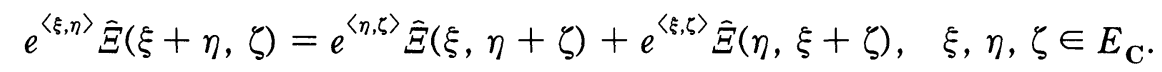

Proof. Since the exponential vectors (4-3) span a dense subspace of $(E), \Xi$ is a derivation if and only if

$$
\Xi\left(\phi_{\xi} \phi_{\eta}\right)=\Xi \phi_{\xi} \cdot \phi_{\eta}+\phi_{\xi} \cdot \Xi \phi_{\eta}, \quad \xi, \eta \in E_{\mathbf{C}}^{*}
$$

With the obvious relation $\phi_{\xi} \phi_{\eta}=e^{\langle\xi, \eta\rangle} \phi_{\xi+\eta}$, we see easily that (5-3) is equivalent to $(5-2)$.

Q.E.D.

Lemma 5.3. Any first order differential operator is a derivation in $\mathscr{L}((E)$, $\left.(E)^{*}\right)$.

Proof. Immediate from Proposition 4.2 and Lemma 5.2.

Q.E.D.

Lemma 5.4. Let $\Xi \in \mathscr{L}\left((E),(E)^{*}\right)$ be a derivation with Fock expansion:

$$
\Xi=\sum_{l, m=0}^{\infty} \Xi_{l, m}\left(\kappa_{l, m}\right)
$$

Then, $\kappa_{l, 0}=0$ for all $l \geq 0$ and

$$
\left\langle\kappa_{l, m+1}, \eta^{\otimes l} \otimes \xi^{\otimes(m+1)}\right\rangle=\left(\begin{array}{c}
l+m \\
l
\end{array}\right)\left\langle\kappa_{l+m, 1},\left(\eta^{\otimes l} \otimes \xi^{\otimes m}\right) \otimes \xi\right\rangle, \quad \xi, \eta \in E_{\mathbf{C}},
$$

for all $l, m \geq 0$.

Proof. By assumption the symbol $\hat{\Xi}$ satisfies $(5-2)$. Then, in view of expansion $(4-5)$ we obtain

$$
\begin{aligned}
& \left(\begin{array}{c}
m+n \\
n
\end{array}\right)\left\langle\kappa_{l, m+n}, \zeta^{\otimes l} \otimes \xi^{\otimes m} \otimes \eta^{\otimes n}\right\rangle \\
& =\left(\begin{array}{c}
l+m \\
m
\end{array}\right)\left\langle\kappa_{l+m, n}, \zeta^{\otimes l} \otimes \xi^{\otimes m} \otimes \eta^{\otimes n}\right\rangle
\end{aligned}
$$




$$
+\left(\begin{array}{c}
l+n \\
n
\end{array}\right)\left\langle\kappa_{l+n, m}, \zeta^{\otimes l} \otimes \eta^{\otimes n} \otimes \xi^{\otimes m}\right\rangle, \quad \xi, \eta, \zeta \in E_{\mathbf{C}}
$$

Then, putting $m=n=0$ in $(5-5)$, we see that $\kappa_{l, 0}=0$ for all $l \geq 0$. We next put $n=1$ and $\eta=\xi$ in (5-5) to obtain

$$
\begin{aligned}
& l !(m+1) !\left\langle\kappa_{l, m+1}, \zeta^{\otimes l} \otimes \xi^{\otimes(m+1)}\right\rangle \\
& =(l+m) !\left\langle\kappa_{l+m, 1},\left(\zeta^{\otimes l} \otimes \xi^{\otimes m}\right) \otimes \xi\right\rangle \\
& \quad+(l+1) ! m !\left\langle\kappa_{l+1, m},\left(\zeta^{\otimes l} \otimes \xi\right) \otimes \xi^{\otimes m}\right\rangle .
\end{aligned}
$$

Applying this argument to the second term successively, we come to

$l !(m+1) !\left\langle\kappa_{l, m+1}, \zeta^{\otimes l} \otimes \xi^{\otimes(m+1)}\right\rangle=(m+1)(l+m) !\left\langle\kappa_{l+m, 1},\left(\zeta^{\otimes l} \otimes \xi^{\otimes m}\right) \otimes \xi\right\rangle$, which completes the proof.

Q.E.D.

Proof of Theorem 5.1. Suppose that we are given a continuous derivation $\Xi$ with Fock expansion as in (5-4). We first introduce a continuous bilinear form $\Omega$ on $E_{\mathrm{C}} \times(E)$ by

$$
\Omega(\xi, \phi)=\sum_{n=0}^{\infty} n !\left\langle\kappa_{n, 1}, f_{n} \otimes \xi\right\rangle, \quad \xi \in E_{\mathbf{C}}, \quad \phi \in(E),
$$

where $\phi(x)=\sum_{n=0}^{\infty}\left\langle: x^{\otimes n}:, f_{n}\right\rangle$. We shall prove the convergence of (5-6). In fact, for any $p, q \geq 0$ we have

$$
\begin{aligned}
\sum_{n=0}^{\infty} n ! & \left|\left\langle\kappa_{n 1}, f_{n} \otimes \xi\right\rangle\right| \\
& \leq \sum_{n=0}^{\infty} n !\left|\kappa_{n, 1}\right|_{-(p+q+1)}\left|f_{n} \otimes \xi\right|_{p+q+1} \\
& \leq\left(\sum_{n=0}^{\infty} n !\left|\kappa_{n, 1}\right|_{-(p+q+1)}^{2}\right)^{1 / 2}\left(\sum_{n=0}^{\infty} n !\left|f_{n}\right|_{p+q+1}^{2}\right)^{1 / 2}|\xi|_{p+q+1} \\
& =|\xi|_{p+q+1}\|\phi\|_{p+q+1}\left(\sum_{n=0}^{\infty} n !\left|\kappa_{n, 1}\right|_{-(p+q+1)}^{2}\right)^{1 / 2} .
\end{aligned}
$$

Since $\Xi \in \mathscr{L}\left((E),(E)^{*}\right)$, there exist $C \geq 0, K \geq 0$ and $p \geq 0$ such that

$$
|\hat{\Xi}(\xi, \eta)| \leq C \exp K\left(|\xi|_{p}^{2}+|\eta|_{p}^{2}\right), \quad \xi, \eta \in E_{\mathbf{C}}
$$

It is proved in [12] that the kernel distributions $\kappa_{l, m}$ of $\Xi$ satisfies

$$
\left|\kappa_{l, m}\right|_{-(p+1)} \leq C\left(l^{l} m^{m}\right)^{-1 / 2}\left(2 e^{3} \delta^{2}\right)^{(l+m) / 2}\left(\frac{\rho^{2 p}}{2}+K\right)^{(l+m) / 2} .
$$


In particular,

$$
\begin{aligned}
\left|\kappa_{n, 1}\right|_{-(p+q+1)} & \leq \rho^{q(n+1)}\left|\kappa_{n, 1}\right|_{-(p+1)} \\
& \leq C \rho^{q(n+1)} n^{-n / 2}\left(2 e^{3} \delta^{2}\right)^{(n+1) / 2}\left(\frac{\rho^{2 p}}{2}+K\right)^{(n+1) / 2} .
\end{aligned}
$$

Therefore,

$$
\sum_{n=0}^{\infty} n !\left|\kappa_{n, l}\right|_{-(p+q+1)}^{2} \leq C^{2} \sum_{n=0}^{\infty} \frac{n !}{n^{n}}\left\{2 e^{3} \delta^{2} \rho^{2 q}\left(\frac{\rho^{2 p}}{2}+K\right)\right\}^{n+1}<\infty
$$

for a sufficiently large $q \geq 0$. In conclusion, we see from (5-7) and (5-8) that

$$
\left|\sum_{n=0}^{\infty} n !\left\langle\kappa_{n, 1}, f_{n} \otimes \xi\right\rangle\right| \leq C_{1}|\xi|_{p+q+1}\|\phi\|_{p+q+1}, \quad \xi \in E_{\mathbf{C}}, \phi \in(E),
$$

for some $C_{1} \geq 0, p \geq 0$ and $q \geq 0$. Therefore $\Omega$ in (5-6) is well defined on $E_{\mathbf{C}} \times$ $(E)$ and becomes a continuous bilinear form.

Let $\tilde{\Phi} \in\left(E_{\mathbf{C}} \otimes(E)\right)^{*}$ be the element corresponding to $\Omega$, namely,

$$
\langle\tilde{\Phi}, \xi \otimes \phi\rangle=\Omega(\xi, \phi), \quad \xi \in E_{\mathbf{C}}, \quad \phi \in(E) .
$$

Let $\Xi^{\prime}$ be the first order differential operator with coefficient $\tilde{\Phi}$. It then follows from Proposition 4.2 that

$$
e^{-\langle\xi, \eta\rangle} \hat{\Xi}^{\prime}(\xi, \eta)=\left\langle\tilde{\Phi}, \xi \otimes \phi_{\xi+\eta}\right\rangle=\Omega\left(\xi, \phi_{\xi+\eta}\right) .
$$

On the other hand, in view of $(5-6)$ we have

$$
\begin{aligned}
\Omega\left(\xi, \phi_{\xi+\eta}\right) & =\sum_{n=0}^{\infty} n !\left\langle\kappa_{n, 1}, \frac{(\xi+\eta)^{\otimes n}}{n !} \otimes \xi\right\rangle \\
& =\sum_{n=0}^{\infty} \sum_{l=0}^{n}\left(\begin{array}{c}
n \\
l
\end{array}\right)\left\langle\kappa_{n, 1}, \quad\left(\eta^{\otimes l} \otimes \xi^{\otimes(n-l)}\right) \otimes \xi\right\rangle \\
& =\sum_{l, m=0}^{\infty}\left(\begin{array}{c}
l+m \\
l
\end{array}\right)\left\langle\kappa_{l+m, 1},\left(\eta^{\otimes l} \otimes \xi^{\otimes m}\right) \otimes \xi\right\rangle .
\end{aligned}
$$

Now, applying the relations:

$$
\left\{\begin{array}{l}
\kappa_{l, 0}=0, \quad l \geq 0, \\
\left\langle\kappa_{l, m+1}, \eta^{\otimes l} \otimes \xi^{\otimes(m+1)}\right\rangle=\left(\begin{array}{c}
l+m \\
l
\end{array}\right)\left\langle\kappa_{l+m, 1},\left(\eta^{\otimes l} \otimes \xi^{\otimes m}\right) \otimes \xi\right\rangle, \quad l, m \geq 0,
\end{array}\right.
$$

which are obtained in Lemma 5.4, we come to 


$$
\Omega\left(\xi, \phi_{\xi+\eta}\right)=\sum_{l, m=0}^{\infty}\left\langle\kappa_{l, m}, \eta^{\otimes l} \otimes \xi^{\otimes m}\right\rangle=e^{-\langle\xi, \eta\rangle} \hat{\Xi}(\xi, \eta)
$$

It follows from (5-9) and (5-10) that

$$
e^{-\langle\xi, \eta\rangle} \hat{\Xi}^{\prime}(\xi, \eta)=e^{-\langle\xi, \eta\rangle} \hat{\Xi}(\xi, \eta), \quad \xi, \eta \in E_{\mathbf{C}},
$$

so that $\Xi=\Xi^{\prime}$. Consequently, $\Xi$ is the first order differential operator with coefficient $\tilde{\Phi}$. The rest of the assertion is now immediate from Lemma 5.3 and Theorem 3.4 .

Q.E.D.

Note that any derivation maps constant functions into zero.

Corollary 5.5. Any continuous derivation on $(E)$ which maps linear functionals into constants is a first order differential operator with constant coefficients and vice versa.

The Gross Laplacian and the number operator are defined respectively as integral kernel operators:

$$
\Delta_{G}=\int_{T \times T} \tau(s, t) \partial_{s} \partial_{t} d s d t, \quad N=\int_{T \times T} \tau(s, t) \partial_{s}^{*} \partial_{t} d s d t
$$

where $\tau \in E^{*} \otimes E$ is given by $\langle\tau, \xi \otimes \eta\rangle=\langle\xi, \eta\rangle, \xi, \eta \in E$.

Corollary 5.6. $\Delta_{G}+N$ is a derivation in $\mathscr{L}((E),(E))$.

\section{REFERENCES}

[1] S. Albeverio, T. Hida, J. Potthoff, M. Röckner and L. Streit, Dirichlet forms in terms of white noise analysis II. Closability and diffusion processes, Rev. Math. Phys., 1 (1990), 313-323.

[2] T. Hida, "Analysis of Brownian Functionals," Carleton Math. Lect. Notes no. 13, Carleton University, Ottawa, 1975.

[3] T. Hida, H.-H. Kuo and N. Obata, Transformations for white noise functionals, J. Funct. Anal., 111 (1993), 259-277.

[4] T. Hida, N. Obata and K. Saitô, Infinite dimensional rotations and Laplacians in terms of white noise calculus, Nagoya Math. J., 128 (1992), 65-93.

[5] T. Hida, J. Potthoff and L. Streit, Dirichlet forms and white noise analysis, Commun. Math. Phys., 116 (1988), 235-245.

[6] N. Ikeda and S. Watanabe, "Stochastic Differential Equations and Diffusion Processes (2nd ed.)," North-Holland, Amsterdam/New York, 1988.

[7] I. Kubo and S. Takenaka, Calculus on Gaussian white noise I-IV, Proc. Japan Acad., 56A (1980), 376-380; 411-416; 57A (1981), 433-437; 58A (1982), 
$186-189$.

[8] I. Kubo and Y. Yokoi, A remark on the space of testing random variables in the white noise calculus, Nagoya Math. J., 115 (1989), 139-149.

[9] H.-H. Kuo, On Laplacian operators of generalized Brownian functionals, in "Stochastic Processes and Their Applications (T. Hida and K. Itô, eds.)," Lect. Notes in Math. Vol. 1203, Springer-Verlag, 1986, pp. 119-128.

[10] P. A. Meyer, "Quantum Probability for Probabilists," Lect. Notes in Math. Vol. 1538, Springer-Verlag, 1993.

[11] N. Obata, Rotation-invariant operators on white noise functionals, Math. Z., 210 (1992), 69-89.

[12] - An analytic characterization of symbols of operators on white noise functionals, J. Math. Soc. Japan, 45 (1993), 421-445.

[13] - Operator calculus on vector-valued white noise functionals, J. Funct. Anal., 121 (1994), 185-232.

[14] - "White Noise Calculus and Fock Space," Lect. Notes in Math. Vol. 1577, Springer-Verlag, 1994.

[15] K. R. Parthasarathy, "An Introduction to Quantum Stochastic Calculus," Birkhäuser, 1992.

Graduate School of Polymathematics

Nagoya University

Nagoya, 464-01 Japan 